\title{
COMPARACIÓN DE NUEVE ALGORITMOS DE MÁQUINAS DE APRENDIZAJE CON APLICACIONES EN LA DETECCIÓN DE ARRECIFE DE CORAL
}

\author{
Eduardo Tusa Jumbo 1', David M. Lane ${ }^{2}$, Neil M. Robertson ${ }^{2}$ \\ Universidad Técnica de Machala (Ecuador) ${ }^{1}$ \\ Heriot-Watt University (Reino Unido) ${ }^{2}$ \\ etusa@utmachala.edu.ec
}

Cómo citar este artículo: Tusa, E., Lane, D. y Robertson, N. (2015) Comparación de nueve algoritmos de máquinas de aprendizaje con aplicaciones en la detección de arrecife de coral. CUMBRES, Revista Científica. 1 (1) 36 - 41

\section{RESUMEN}

Este trabajo se enfoca en la comparación de nueve algoritmos de máquinas de aprendizaje para el desarrollo de un detector de coral. Este detector consta de una parte de extracción de vectores característicos, la cual se realiza con filtros Gabor Wavelets; y una parte de clasificación de vectores que usa máquinas de aprendizaje, basado en Redes Neuronales. Se realizó la comparación de la precisión y el tiempo de ejecución de nueve algoritmos de máquinas de aprendizaje, cuyo resultado fue la selección del algoritmo de Árboles de Decisión. Se implementó un banco de filtros Gabor Wavelets utilizando C++ y la librería OpenCV. Se utilizó una base de datos de 621 imágenes de corales de Belice (110 imágenes de entrenamiento y 511 imágenes de prueba). Nuestro detector de coral posee un tiempo de ejecución de 70 ms con $70 \%$ de precisión.

Palabras clave: OpenCV, arrecife de coral, máquinas de aprendizaje, filtros Gabor Wavelets, descriptores de textura.

\begin{abstract}
This work focuses on the comparison of nine machine learning algorithms for developing a coral reef detector. This detector has two parts: feature extraction that uses Gabor Wavelet filters, and feature classification that uses machine learning based on Neural Networks. We compare the accuracy and running time of nine machine learning algorithms,
\end{abstract}

whose result was the selection of the Decision Trees algorithm. We implement the bank of Gabor Wavelets filters using $\mathrm{C}_{+}$and the OpenCV library. We use a database of 621 images of coral reef in Belize (110 images for training and 511 images for testing). Our coral detector performs $70 \mathrm{~ms}$ of running time with $70 \%$ of accuracy.

Keywords: OpenCV, coral reef, machine learning, Gabor Wavelet filters, texture descriptors.

\section{INTRODUCCIÓN}

La restauración del arrecife de coral de manera convencional envuelve condiciones extremas para los buceadores voluntarios que trasplantan fragmentos perdidos de arrecife hacia un marco de arrecife de coral más grande. Lophelia pertusa constituye una de las construcciones de especies de coral más importantes en el mundo. No obstante, su ubicación genera limitaciones a la intervención humana debido a las bajas temperaturas y la profundidad del mar que es alrededor de 200 metros.

El proyecto Coralbot es una propuesta reciente para la reparación autónoma de los arrecifes de coral, con la finalidad de conservar los ecosistemas oceánicos; los mismos que son vitales para la pesca comercial, el turismo y la supervivencia de otras especies. La idea consiste en combinar vehículos autónomos submarinos (autonomous underwater vehicle, AUV, por sus siglas en inglés) con inteligencia de enjambre. Este tipo de inteligencia reproduce 
el comportamiento de organismos como abejas, termitas o avispas que actúan en grupo desarrollando tareas complejas utilizando reglas sencillas. De esta forma, el equipo de AUVs es desplegado para reconocer arrecifes de coral y ejecutar tareas de restauración.

La presente investigación propone la implementación de un detector de coral de desempeño rápido para que sea incorporado en el robot Coralbot. En este ambiente se requiere una respuesta de posicionamiento inmediato del AUV frente al arrecife de coral; de otro modo, éste perdería su orientación, lo que causaría daños al coral o a sí mismo (Coralbot, 2013).

Nos enfocamos en la obtención de los vectores característicos de textura, como lo realizó (Purser et al., 2009), utilizando filtros de Gabor Wavelets que fueron implementados usando C++ y la (Librería OpenCV, 2008). Sin embargo, se hizo un estudio del desempeño del algoritmo de clasificación de estos descriptores de textura debido a que las Redes Neuronales utilizadas por (Purser et al., 2009) consumían mucho tiempo de cómputo. Por este motivo, se evaluó la precisión y el tiempo de ejecución de nueve algoritmos de máquinas de aprendizaje. El algoritmo de Árboles de Decisión sustituye a las Redes Neuronales utilizadas por (Purser et al., 2009).

Una cantidad considerable de literatura ha sido publicada sobre la extracción de descriptores característicos. Generalmente, la imagen es transformada en un conjunto de vectores característicos, de modo que las diferentes regiones o formas deseadas son descritas cuantitativamente mediante sus propiedades: intensidad de color, información de textura, datos del espacio, señales de borde.

El tipo de características utilizadas en la mayoría de los artículos científicos se basan en la información de color y textura. Siguiendo esta tendencia, (Stought et al., 2012) proponen el uso de características de color denominadas funciones cuantiles y descriptores de textura usando la transformada de características de escala invariante (SIFT, por sus siglas en inglés). Las características de color proporcionan buenos resultados, mientras las características SIFT fueron sensibles al ruido.

Las características de texturas son generadas usando una versión rotacional invariante de los Patrones Binarios Locales (LBP), por sus siglas en inglés para generar un histograma de diez componentes. Para (Marcos et al., 2008), los valores de la media, el matiz y la saturación para la porción de la imagen; o los pesos mayores de los canales rojo y verde. Éstos son obtenidos mediante la comparación en el espacio normalizado de color rojo y verde, de los histogramas de las porciones de la imagen con aquellos obtenidos para los cuatro grupos de colores que ocurren en el ambiente del arrecife.

La información de color es también incluida mediante la aplicación de filtros a cada canal en el espacio de color Lab y apilando los vectores de respuesta del filtro. (Beijbom et al., 2012) utiliza un banco del filtro rotacional invariante (el banco del filtro de respuesta máxima (MR)) para datos de textura.

Según (Johnson et al., 2006) generan 60 características visuales: 12 son la media y la desviación estándar de los canales RGB y HSV respectivamente. Los 48 restantes son obtenidos mediante la convolución de la región con los filtros Gabor Wavelets a seis escalas y cuatro orientaciones, tomando la media y la desviación estándar de los resultados para cada escala y orientación.

Para comparar un sistema de visión de computadoras con el uso de métodos manuales utiliza un cuadrante de 15 puntos, cuadrante de 100 puntos y un marco de mapeo. (Purser et al., 2009) calcula 15 máscaras diferentemente orientadas y espaciadas con el fin de producir un conjunto de 30 características de texturas.

Puesto que la información de color es susceptible a la escasa iluminación del fondo marino, se decidió explotar las características de texturas a través del uso del filtro Gabor Wavelets que utiliza (Purser et al., 2009) y (Johnson et al., 2006); pero utilizando siete escalas y cinco orientaciones.

El diseño del algoritmo orientado a la discriminación de las clases, coral o no coral, puede ser direccionado de diferentes maneras. Se propone la utilización de algoritmos de máquinas de aprendizaje supervisado. Esto consiste en tomar un vector característico por cada píxel de la imagen y asignarle su clase, coral o no coral. La asignación de un vector característico se ajusta a un modelo de predicción derivado de los datos de entrenamiento.

En el trabajo de (Purser et al., 2009) se realiza la detección de coral utilizando Redes Neuronales. A pesar de que los resultados son satisfactorios, el algoritmo consume mucho tiempo de cómputo para la detección de coral. Este tiempo excesivo de procesamiento podría llevar al robot a enfrentar condiciones catastróficas.

Por esta razón, esta investigación evaluó el tiempo de ejecución del desempeño de nueve algoritmos de máquinas de aprendizaje para seleccionar el más rápido, estos son: 
1) Árboles de Decisión (DTR), Árboles Aleatorios (RTR), Árboles extremadamente aleatorios (ERT), Boosting (BOO), Gradiente de Árboles Boosting (GBT), Clasificador Normal Bayes (NBA), Maximización de la Esperanza (EMA), Redes Neuronales (MLP), Máquinas de Vectores de Soporte (SVM). El criterio utilizado para la selección de estos algoritmos radica en que se encuentran implementados en $\mathrm{C}++, \mathrm{y}$ disponibles en la (Librería OpenCV, 2008).

A continuación discutiremos los materiales y métodos donde se resumen las características del algoritmo y los equipos empleados para la evaluación. La siguiente sección presenta los resultados de la evaluación de cada algoritmo. Finalmente, se presentan las conclusiones.

\section{MATERIALES Y MÉTODOS}

El detector de coral toma una imagen del conjunto de imágenes de prueba y sigue los siguientes pasos Figura 1. (Diagrama de bloques del Detector de Coral: A. Pre-procesamiento, B. Extracción de características de textura, C. Clasificación y D. Pos-procesamiento):

- Pre-procesamiento: La imagen se lleva a escala de grises para normalizar el valor del píxel.

- Extracción de características de textura: Los descriptores característicos de textura se extraen mediante la convolución de la imagen con un banco de filtros Gabor Wavelets. Siguiendo este procedimiento, a cada píxel de la imagen se le asocia un vector característico. - Clasificación: Se realiza un proceso de discriminación entre la clase coral y no coral mediante un aprendizaje supervisado de máquinas haciendo uso de los nueve algoritmos mencionados anteriormente.

- Pos-procesamiento: Los falsos positivos y los falsos negativos son removidos mediante la selección de los contornos más grandes.

La base de datos contiene 621 imágenes provenientes del arrecife de coral ubicado en la costa de Belice, y fue recogida por la Dra. Lisa Carne. Esta base de datos de imágenes de arrecifes de coral fue elaborada para fines experimentales y el tamaño estándar de las fotos fue seleccionado a ser 1024 × 768 píxeles. Además, se encuentra disponible en el repositorio del Laboratorio de Visión de Computadoras de la Universidad Heriot-Watt.

Este conjunto de imágenes se divide en 110 imágenes para entrenamiento y 511 imágenes para prueba. Sobre cada imagen, el experto seleccionó regiones de interés de la imagen con la forma de un polígono, para separar las regiones que pertenecen al coral, de las regiones que no pertenecen al coral, asignándoles los valores +1 y -1 , respectivamente Figura 2. (Las regiones de interés de la imagen está conformada por polígonos de n puntos para una clase de coral, y m puntos para una clase que no es coral), seleccionando (n) puntos para la clase coral, y (m) puntos para la clase no coral. Los elementos, en estas regiones seleccionadas, se almacenan en un vector, en donde se registran las coordenadas de los píxeles dentro de estas regiones.

Figura 1. Diagrama de bloques del Detector de Coral: A. Pre-procesamiento, B. Extracción de características de textura, C. Clasificación y D. Pos-procesamiento

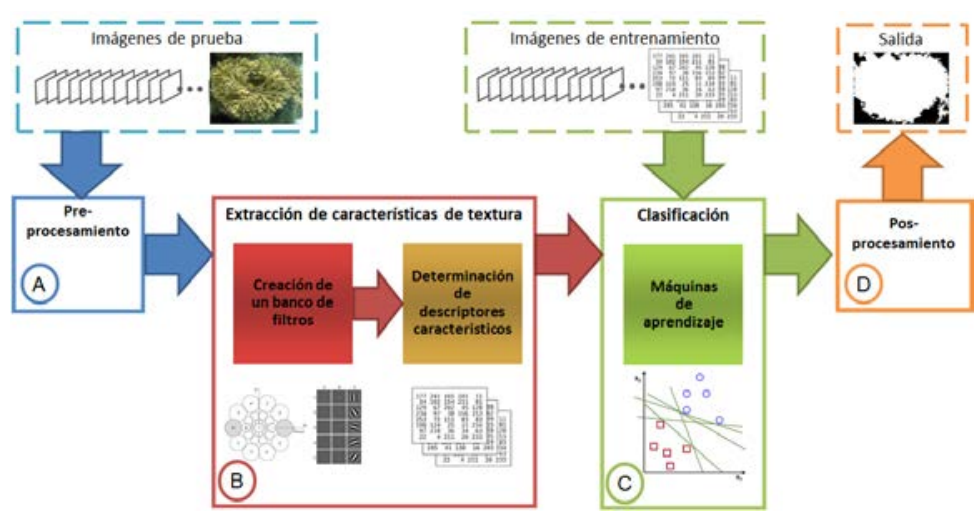

Elaborado por: (Tusa et al., 2014) 
Figura 2. Las regiones de interés de la imagen está conformada por polígonos de $n$ puntos para una clase de coral, y m puntos para una clase que no es coral

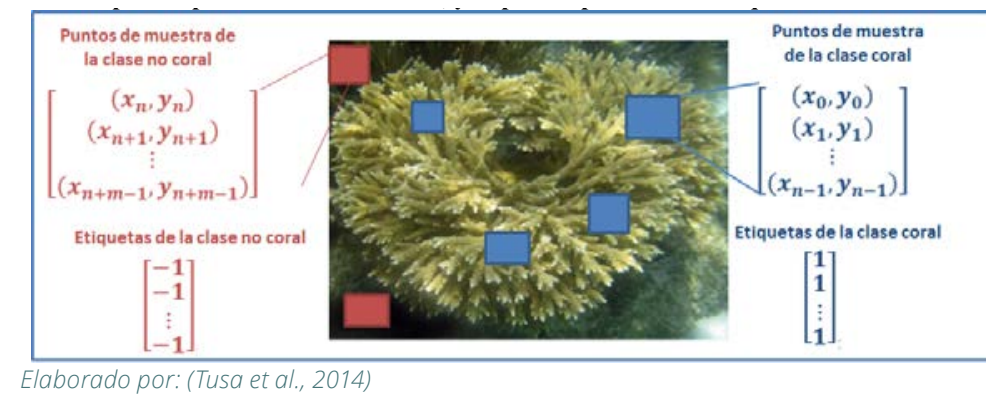

En el dominio espacial, un filtro Gabor 2D es el resultado de una función Gaussiana modulada por una onda sinusoidal. La expresión matemática para este filtro se describe en (1)

$$
g(\mathrm{x}, \mathrm{y})=\mathrm{e}^{-\left(\frac{\left(x-x_{0}\right)^{2}}{2 \sigma_{x}^{2}}+\frac{\left(y-y_{0}\right)^{2}}{2 \sigma_{y}^{2}}\right)} e^{-i k\left(x-x_{0}\right)}(1)
$$

Donde es el centro de la onda Gaussiana, y son las varianzas de la Gaussiana a lo largo de los ejes x y y, respectivamente; es la frecuencia espacial de una onda en el plano complejo con la onda normal a lo largo del eje $x, y$ es la longitud de onda.

Se trabaja con filtros Gabor Wavelets que son funciones similares a sí mismas. Si se considera a la función $g(x, y)$ como la Gabor Wavelets matriz, entonces un banco de filtros similares a sí mismos puede ser creado mediante escalamientos y rotaciones de la función $g(x, y)$ a través de las expresiones (2), (3) y (4).

$$
\begin{gathered}
g_{m n}=g\left(x^{\prime}, \mathrm{y}^{\prime}\right) \\
x^{\prime}=a^{-m}\left(x \cos \Theta_{n}+y \sin \Theta_{n}\right) \\
y^{\prime}=a^{-m}\left(-x \sin \Theta_{n}+y \cos \Theta_{n}\right)
\end{gathered}
$$

El diseño del diccionario conformado por los filtros Gabor Wavelets utiliza 7 escalas y 5 orientaciones diferentes.

Los experimentos de este trabajo consistieron en comparar los nueve algoritmos: Árboles de Decisión (DTR), Árboles Aleatorios (RTR), Árboles extremadamente aleatorios (ERT), Boosting (BOO), Gradiente de Árboles Boosting (GBT), Clasificador Normal Bayes (NBA), Maximización de la Esperanza (EMA), Redes Neuronales (MLP), Máquinas de Vectores de Soporte (SVM); disponibles en la (Librería OpenCV, 2008). Se usó una base de datos de 621 imágenes de los arrecifes de coral de Belice, con 110 imágenes para entrenamiento y 511 imágenes para prueba.
En estos experimentos se utiliza una laptop ASUS G55V con procesador Intel Core i7 - 3610QM a 2.30 GHz, con memoria RAM de 12 GB, con una tarjeta gráfica NVIDIA GEFORCE GTX 660M 2GB y con sistema operativo UBUNTU. Esto proporciona un ordenador con el triple del número de núcleos que el computador utilizado por (Purser et al., 2009), el cual es una laptop Dell M1530 con un procesador Intel Core2 Duo a 2.4 GHz con sistema operativo LINUX.

Los expertos seleccionaron regiones de interés de las 110 imágenes de entrenamiento y generaron 6.800.071 vectores característicos, para el entrenamiento de los algoritmos clasificadores de máquinas de aprendizaje, mencionadas anteriormente. La Figura 3. (Tiempo de ejecución en segundos de los nueve clasificadores supervisados de máquinas) resume las diferentes métricas utilizadas para comparar los algoritmos clasificadores: Tiempo de ejecución y precisión.

\section{RESULTADOS Y DISCUSIÓN}

El resultado de estos experimentos de comparación dio como algoritmo más rápido, el Árbol de Decisión (DTR) al clasificar una imagen de 1024 × 768 píxeles en un tiempo de ejecución de 70 ms. Puesto que se utilizaron 511 imágenes de prueba, se promediaron aritméticamente los tiempos empleados por cada algoritmo para clasificar cada imagen. El registro de tiempos fue realizado en el programa C++ a través de la función tic-toc. El tiempo de todos los algoritmos se puede apreciar en la Figura 3. (Tiempo de ejecución en segundos de los nueve clasificadores supervisados de máquinas).

La precisión mide la tasa de predicciones correctas del número total de casos evaluados. La ecuación (5) describe el cálculo de esta métrica 
Figura 3. Tiempo de ejecución en segundos de los nueve clasificadores supervisados de máquinas

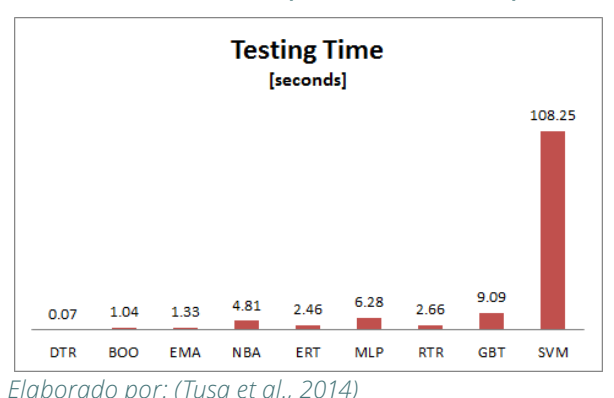

donde PV es el número de positivos verdaderos, NV es el número de negativos verdaderos, PF es el número de positivos falsos y NF es el número de negativos falsos.

$$
\text { precisión }=\frac{P V+N V}{P V+N V+P F+N F}(5)
$$

Las imágenes de referencia provienen de las 511 imágenes de entrenamiento que fueron muestreadas por los expertos como se señaló en la Figura 2. (Las regiones de interés de la imagen está conformada por polígonos de $n$ puntos para una clase de coral, y m puntos para una clase que no es coral).

Por lo tanto, el valor de precisión para cada algoritmo viene del total de píxeles muestreados de las 511 imágenes.

En la Figura 4. (Precisión de los nueve clasificadores supervisados de máquinas) se aprecia que ERT, MLP, GBT y BOO sobrepasan el 70\% de precisión. DTR, NBA y EMA poseen precisiones que no sobrepasan el 60\%. SVM no produce una clasificación notoria entre los nueve clasificadores.

La Figura 5. (Resultados de la detección del coral Staghorn) resume los resultados obtenidos para la tarea de clasificación del coral Staghorn. En la (Figura 5a) se presenta la
Figura 4. Precisión de los nueve clasificadores supervisados de máquinas

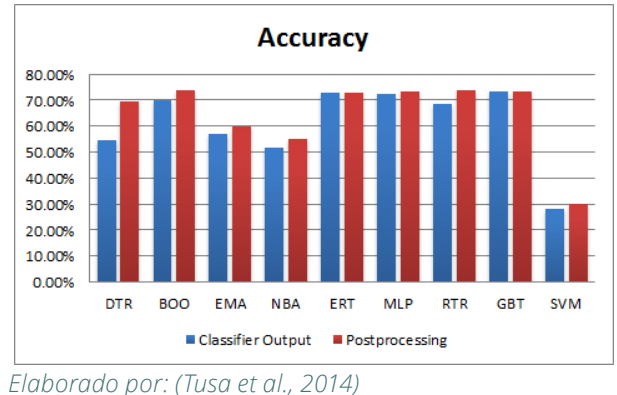

imagen de entrada, mientras que las Figuras restantes, presentan los resultados de los algoritmos de clasificación mencionados anterior. La clase no coral o plano de fondo, se ha coloreado de blanco con el fin de contrastar con lo que el sistema reconoce como coral. Las (Figuras 5b y 5c) presentan los resultados de los algoritmos DTR y BOO, respectivamente. Se aprecia una ubicación satisfactoria de la región que corresponde al coral dentro de la escena. El algoritmo de EMA (ver Figura 5d) pierde levemente algunas detecciones positivas verdaderas. No obstante, el NBA (ver Figura 5e) incrementa notoriamente el número de falsos negativos, enviando píxeles de coral al plano de fondo. Las (Figuras $5 f$ y $5 g$ ) que corresponden a los algoritmos ETR y MLP, respectivamente; presentan una alta presencia de falsos positivos, es decir; píxeles del plano de fondo considerados como arrecifes de coral. El algoritmo RTR (ver Figura 5h) muestra resultados similares a DTR y a BOO; sin embargo, algunos falsos positivos se filtran en el plano de fondo. La (Figura 5i) corresponde al algoritmo de GBT, el cual presenta dificultades para reconocer al buceador como plano de fondo. Finalmente, la (ver Figura 5j) muestra el resultado del algoritmo SVM. La imagen presenta una pequeña presencia de coral con un abundante plano de fondo detectado como coral. Esto dificulta focalizar un sector específico de arrecife de coral en la escena.

Figura 5. Resultados de la detección del coral Staghorn

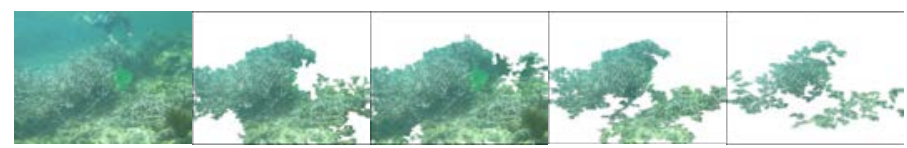

(a) INPUT

(b) DTR

(c) $\mathrm{BOO}$

(d) EMA

(e) NBA

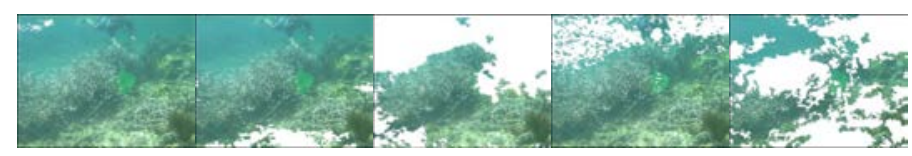

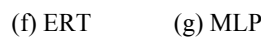

Elaborado por: (Tusa et al., 2014) (h) RTR

(i) GBT

(j) SVM 
La Tabla 1. (Tiempo de ejecución y Precisión de cada algoritmo de clasificación supervisado) resume el tiempo de ejecución en segundos realizado por cada algoritmo evaluado en este proyecto, y el porcentaje en la precisión para la detección de arrecife de coral.

Tabla 1. Tiempo de ejecución y Precisión de cada algoritmo de clasificación supervisado

\begin{tabular}{|c|l|c|c|}
\hline No. & Algoritmo de clasificación supervisado & $\begin{array}{c}\text { Tiempo de } \\
\text { ejecución } \\
\text { en segundos }\end{array}$ & $\begin{array}{c}\text { Precisión en } \\
\text { porcentaje }\end{array}$ \\
\hline $\mathbf{1}$ & Árboles de Decisión (DTR) & 0,07 & $69,58 \%$ \\
\hline $\mathbf{2}$ & Árboles Aleatorios (RTR) & 2,66 & $73,82 \%$ \\
\hline $\mathbf{3}$ & Árboles extremadamente aleatorios (ERT) & 2,46 & $60,17 \%$ \\
\hline $\mathbf{4}$ & Boosting (BOO) & 1,04 & $55,39 \%$ \\
\hline $\mathbf{5}$ & Gradiente de Árboles Boosting (GBT) & 9,09 & $73,23 \%$ \\
\hline $\mathbf{6}$ & Clasificador Normal Bayes (NBA) & 4,81 & $73,47 \%$ \\
\hline $\mathbf{7}$ & Maximización de la Esperanza (EMA) & 1,33 & $74,00 \%$ \\
\hline $\mathbf{8}$ & Redes Neuronales (MLP) & 6,38 & $73,54 \%$ \\
\hline $\mathbf{9}$ & Máquinas de Vectores de Soporte (SVM) & 108,25 & $30,24 \%$ \\
\hline
\end{tabular}

Elaborado por: (Tusa et al., 2014)

\section{CONCLUSIONES}

1. Este artículo presenta la comparación de nueve algoritmos para el desarrollo de un detector de coral que clasifica entre dos clases: la clase que pertenece al coral y el plano de fondo que no pertenece al coral.

2. El algoritmo de aprendizaje supervisado se entrena con vectores característicos de textura generados por un banco de filtros Gabor Wavelets.

3. Nueve clasificadores fueron evaluados mediante 2 métricas.

4. Los clasificadores: Árboles extremadamente aleatorios, Gradiente de Árboles Boosting, Redes Neuronales y Máquinas de Vectores de Soporte; mostraron dificultades para discriminar las clases.

5. En contraste, los clasificadores: Árboles de Decisión, Boosting, Maximización de la Esperanza, Clasificador Normal Bayes y Árboles Aleatorios; presentaron una clasificación visual prometedora.

6. El algoritmo de Árboles de Decisión (DTR) presentó el menor tiempo de ejecución con la más alta precisión. Por esta razón, se recomienda la utilización del algoritmo de Árboles de Decisión en el detector de coral del robot Coralbot.

\section{REFERENCIAS BIBLIOGRÁFICAS}

- Beijbom, O., Edmunds, P., Kline, D., Mitchell, G. \& Kriegman, D. (2012). Automated Annotation of Coral Reef Survey Images. Proceedings of the 2012 IEEE Conference on Computer Vision and Pattern Recognition (CVPR).

- Bradski, G., \& Kaehler, A. (2008). Learning OpenCV. Cambridge: Computer Vision with the OpenCV Library.

- Coralbot, T. (2013). About coral reefs and current approaches for restoration. Obtenido de Coralbot Web site: http://www.coralbots.org/.

- Johnson, R., Kumar, S., \& Willams, S. (2006). Segmentation and classification of coral for oceanographic surveys. A semi-supervised machine learning approach. Proceedings of OCEANS 2006 - Asia Pacific.

- Marcos, M., David, L., Peñaflor, E., Ticzon, V. \& Soriano, M. (2008). Automated benthic counting of living and non-living components in Ngedarrak Reef, Palau via subsurface underwater video. Environmental Monitoring and Assessment.

- Purser, A., Bergmann, M., Lundälv, T., Ontrup, J. \& Nattkemper, T. (2009). Use of machine-learning algorithms for the automated detection of cold-water coral habitats. A pilot study. 397(3354/meps08154).

- Stough, J., Greer, L. \& Matt, B. (2012). Texture and Color Distribution-based Classification for Live Coral Detection. Proceedings of the 12th International Coral Reef Symposium. 